\title{
Test sample selection by preschool children: Honoring diversity
}

\author{
ELIZABETH F. SHIPLEY and BARBARA SHEPPERSON \\ University of Pennsylvania, Philadelphia, Pennsylvania
}

\begin{abstract}
In a test of an inductive inference, preschool children's selection of objects was examined. In Experiment 1, 4-year-olds selected diverse objects first in sequential selections; in Experiment 2, adults and 4-year-olds, but not 3-year-olds, made similar selections under the same conditions. A defective object led subjects in all age groups to test a similar object. In Experiment 3, 4-year-olds chose to test a pair of dissimilar objects rather than a pair of similar objects, but 3-year-olds did not. Three-year-olds' selections were independent of diversity. In Experiment 4, we attempted to emphasize the diversity of objects for 3-year-olds. Their first task was to select an object that was the same as or different from a target object. The subjects responded correctly in this task but did not prefer to test diverse objects. Experiment 5 showed that neither 3- nor 4-year-olds have a bias to select nondiverse objects in a nontest context. The findings indicate that children as young as 4 years old value diverse evidence in induction.
\end{abstract}

It is intuitively compelling that more diverse evidence leads to a stronger conclusion. A restaurant reviewer samples different dishes on different visits before recommending a restaurant. A political candidate needs to express her opinion on a variety of issues. Philosophers have long argued for the importance of diversity in evidence. (See the summary in Heit, Hahn, \& Feeney, 2004.) However, experimental tests of the effects of diversity have produced mixed findings.

Osherson, Smith, Wilkie, López, and Shafir (1990) inaugurated contemporary work on diversity with adults through the use of an argument strength experimental procedure and a model of inductive inferences that can account for 11 phenomena, including diversity. In the argument strength paradigm, a subject is told of premise categories that possess an unfamiliar property and a conclusion category that possesses the same property, and is asked to rate the strength of an argument. Some arguments had diverse premises (e.g., dogs and cats), and others had similar categories (e.g., dogs and wolves). Arguments with diverse premises were rated as stronger, indicating that diverse evidence offers more support than similar evidence for an inductive inference. Osherson et al. used arguments with unfamiliar properties. They found evidence for diversity for both general arguments, in which the conclusion category includes the premise categories (e.g., animals),

Some of this work was reported at the 44th Annual Meeting of the Psychonomic Society, Vancouver, 2003. We thank the children, teachers, and parents of the Avon Grove Nazarene Day Care Center for their generous cooperation. Ulrike Hahn and two anonymous reviewers made valuable comments on an earlier version of this article. We are very grateful to Dr. Hahn for suggesting Experiment 5. B.S. is now at the Educational Research and Development Center of the University of Delaware, Newark. Correspondence concerning this article should be addressed to E. F. Shipley, Department of Psychology, University of Pennsylvania, 3720 Walnut Street, Philadelphia, PA 19104-6196 (e-mail: liz@psych.upenn.edu). and specific arguments, in which the premise categories and the conclusion category are all at the same level in a class inclusion category (e.g., horses).

In most subsequent work on the effect of diversity on argument strength, a forced-choice argument comparison task was used. López, Atran, Coley, and Medin (1997), using have a disease as the property, found that American college students favored arguments with diverse premises but adult Itzaj Mayan subjects did not. Under some conditions, the Itzaj Mayan subjects judged the argument with the biologically more similar premises as stronger.

Choi, Nisbett, and Smith (1997) used biological and behavioral properties in the arguments. They found diversity effects in general arguments but not in specific arguments with American college students. Proffitt, Coley, and Medin (2000) found little or no effect of diversity with American tree experts. Finally, Medin, Coley, Storms, and Hayes (2003), who used an argument rating procedure, found that causal relations among premise categories could cancel the effect of diversity.

Children's reactions to diverse evidence have been even more mixed. Carey (1985) told 6-year-old children a property of two diverse animates, such as dogs and bees, and asked if other animates possessed the property. She found that the willingness of 6 -year-olds to project a property from one category to another was not enhanced by learning that a less similar category also had the property.

López, Gelman, Gutheil, and Smith (1992) used a simplified version of the forced-choice argument strength paradigm with 5- and 8-year-olds and with unfamiliar properties. The five-year-olds were indifferent to diversity. For the 8-year-olds, general arguments with diverse premises were stronger than general arguments with similar premises. However, diversity of premises had no effect on the strength of specific arguments.

Heit and Hahn (2001) used a somewhat different type of inference task, in which the subject had to decide which 
person would like a novel object: a person who already possessed a diverse set of objects or one who possessed a uniform set. Two sets of objects, corresponding to the two alternatives, were presented. Heit and Hahn found that diversity effects in 5-year-olds varied with the properties. Five-year-olds were sensitive to diversity when the judgment involved individuals and surface properties (e.g., the colors of chocolate bar wrappers). Alternatively, when the property was an internal state (e.g., nuts inside chocolate bars), the children were indifferent to diversity. These findings are important because they demonstrate sensitivity to diversity by young children and raise questions about the use of unseen properties in reasoning tasks. However, it is not clear that they demonstrate heightened effectiveness of diverse evidence in a category-based induction task. The results could also be accounted for by the children's preference to classify novel objects that are unlike members of either alternative set with a set of perceptually diverse objects rather than with a set of perceptually homogeneous objects. We see both explanations as possible and as compatible with the children's justifications.

Lo, Sides, Rozelle, and Osherson (2002) found that diversity effects with preschool children varied with property. In argument strength induction tasks with two premise categories, inductive inferences were stronger when the premises were judged as less likely to share the property, independent of the diversity of the premises.

Hayes, Goodhew, Heit, and Gillan (2003) used a more ecologically valid task-learning about the shape of the earth-and demonstrated with 6-year-olds that diverse information facilitated the acquisition of a mental model of a spherical earth.

Shipley and Shepperson (2004) worked with 4-yearolds using a forced-choice argument strength procedure (based on López et al., 1992) and found that the effect of premise diversity depended on the wording of the question to the child. The children were told that dogs and cats have alpha inside and dogs and wolves have beta inside. They were then asked about "all animals" with questions using one of two wordings: aligned wording, in which the same category was mentioned first in each alternative (e.g., "Do you think all animals have inside what dogs and wolves have inside, or what dogs and cats have inside?"), and nonaligned wording, in which different categories were mentioned first in the two alternatives (e.g., "Do you think all animals have inside what wolves and dogs have inside, or what cats and dogs have inside?"). With nonaligned wording, the children strongly preferred the argument with the more similar premise categories. Only when the children heard aligned wording did they find arguments with diverse premises stronger. López et al. (1992), who found no diversity effects with 5 -year-olds, had used nonaligned wording in tests of diversity. However, Lo et al. (2002), who used aligned wording, found evidence that diversity facilitated induction in preschool children.

Shipley and Shepperson (2004) attributed the difference in performance with different types of question wording to the difficulty of processing the less conventional, nonaligned wording (see Clark \& Clark, 1977, for a discus- sion of the effect of presentation of old information before new information) and to subjects' defaulting to a similarity judgment when confused (see, e.g., Kemler Nelson, Frankenfield, Morris, \& Blair, 2000). This interpretation suggests that another, less verbal, procedure might lead to more consistent facilitation of induction by diversity.

López (1995) gave American college students a singlepremise general argument and asked them to select an additional category to be tested to evaluate the argument. The students selected the more dissimilar category as the additional premise category and explicitly mentioned dissimilarity when asked to justify their choices. Another population (adult members of a preindustrial Mayan society) did not honor diversity in argument strength tasks but did when asked about selection of seeds for tests (López et al., 1997). They preferred to select samples of seeds from different sources rather than several samples from a single bag. This pattern suggests that these subjects found evidence selection a simpler task than comparison of two arguments. Indeed, evidence selection is something adults do constantly in tasks ranging from deciding which state produces the best tomatoes to deciding which kind of printer produces the cleanest copies. It is a more ecologically valid explanation of how diversity guides our choices than are argument strength procedures.

\section{Summary of Prior Work}

Three experimental techniques have been used to study the effect of diverse evidence on induction in adults. The first is the argument strength rating paradigm, in which the subject indicates the strength of an argument by assigning a number to it and the diversity of the premises is varied (Medin et al., 2003; Osherson et al., 1990). When subjects judge arguments with diverse premises as stronger, this indicates that they conceive of diverse evidence as offering more effective support for inductive inferences. The second experimental technique is the forced-choice argument strength paradigm, in which two arguments, one with more diverse premises than the other, are presented and the subject must select the stronger argument (see, e.g., López et al., 1997). Again, selection of the argument with the more diverse premises indicates the subject's belief in the greater effectiveness of diverse evidence in support of inductive inferences. The third technique is a data selection paradigm, in which a premise category and a conclusion category are given and the subject must select which of a set of additional categories should be tested to evaluate the conclusion (López, 1995). The selection of a category that is least similar to the premise category is an indication that adults prefer diverse data to evaluate an inductive inference.

In brief, for the most part studies of diversity and induction have shown that adults consider inferences supported by diverse evidence to be stronger than those supported by similar evidence. The only study on evidence selection revealed that adults select diverse evidence to evaluate an inductive inference.

Somewhat different procedures have been used with children. First, a rating scale technique is difficult to use 
with children; in the study of the effect of diversity on induction, its analogue is to provide some subjects with diverse information and others with similar information and to compare the effectiveness of the different types of information on the conclusions made by the different groups. Diverse evidence apparently offers stronger support for a conclusion (Hayes et al., 2003). Second, the two-alternative forced choice, as developed by López et al. (1992), has been used most often with children to study argument strength. It has yielded mixed results with preschool children (Lo et al., 2002; López et al., 1992; Shipley \& Shepperson, 2004). A different type of forcedchoice argument strength procedure is to present two inductive inferences and a single object and to ask the child to decide which inference applies to the object (Heit \& Hahn, 2001). Finally, the issue of a preference for diverse information (López, 1995), as opposed to the effectiveness of diverse information, has not been examined with children.

We developed a test of children's appreciation of diversity that does not involve argument strength ratings or comparisons. To determine whether or not preschool children seek diverse evidence for testing, we had subjects select evidence to evaluate a hypothesis. This should be a more direct way to study the effect of diversity on induction with children, as it apparently is for adults (López et al., 1997). The use of only three categories, the lack of category labels, and the use of 3-D objects should all simplify the task for children.

In four experiments, each child's task was to help a puppet decide what kinds of toys would make good party favors. The experimenter explained that the puppet had been to a party favor store that had many kinds of favors and couldn't decide which kinds to get for his party. So, the puppet bought some of each of several different kinds of favors and was going to go back and get more of the best ones. He needed the child's help to decide which would be good party favors. Each of the different kinds of toys had a canonical movement or action (e.g., cars roll, whistles can be blown). Each sample set of toys presented to the child consisted of two subsets that differed in a surface property (e.g., color). The child's task was to select from the samples the objects to be tested to evaluate the toys.

The inductive inferences involved going from a sample of toys of a given kind (e.g., several whistles) to the category of that kind of toy (e.g., whistles). The projected property, expressed as "would make a good party favor," was an attempt to express the concept "perform its canonical function" in a child-friendly way.

Experiment 1 demonstrates that 4-year-olds select diverse evidence to test the functioning of various types of toys. Experiment 2 includes both 3 -year-old subjects (to examine the lower limits of sensitivity to diversity) and adults (to validate our procedure as a test for the preference for diverse evidence, as was found with adults by López, 1995). In an attempt to support our claim that the subjects were attempting to evaluate toys as good party favors, we also included defective objects in Experiment 2.
A differential response to defective toys would indicate the subjects' awareness of what constitutes an inadequate party favor. Finally, Experiment 2 corrects for the possibility that the instructions to the children in the first experiment may have taught them that subclasses differ and, hence, that instances of both subclasses should be tested. In Experiment 3, in which 3- and 4-year-olds served as subjects, on each trial the children made a single choice to test either a diverse pair or an identical pair. This procedure removes the possibility that a sequential response bias had determined the choices in the first two experiments. Experiment 4 ensured that 3 -year-olds could distinguish the subclasses. Finally, in Experiment 5, which required children to put away toys one by one, we sought evidence for a bias for diverse objects in a nontest task.

\section{EXPERIMENT 1}

In the initial experiment, the children selected, one by one from the sample, the objects to be tested. The initial selection of two toys from different subsets was considered a preference for diverse evidence.

\section{Method}

Subjects. Twelve 4-year-olds ( 5 girls and 7 boys; age range, $4 ; 1-$ $4 ; 4$ years, $M=4 ; 3$ ) participated in the experiment. All attended a preschool serving a middle-class population.

Stimuli. Eight horns - four of them red and making a sound when blown and four of them green and making no sound when blownwere used to introduce the task. Five sets of 20 toys, each of which consisted of two homogeneous subsets of 10 objects, were used on the test trials. The sets consisted of (1) yoyos, 10 felt covered and 10 leather covered; (2) blowers (in the form of paper tubes that unroll when blown), 10 with woolen braids and 10 with pompons on the ends; (3) miniature animals, 10 of them cows and 10 of them pigs; (4) clickers, 10 decorated with felt and 10 decorated with wool; and (5) cars, 10 red and 10 blue.

Procedure. The experimenter and a child sat together on the floor in a quiet area of the school. The experimenter introduced a puppet and explained that the puppet needed to evaluate various kinds of toys. The puppet planned to give a party and wanted to get good party favors for his friends. The experimenter then presented two boxes of horns: one box of four red horns that worked and one box of four green ones that did not work. After testing all the horns, she explained that horns would not be good party favors because some do not work.

Then, the five sets of potential party favors were presented one at a time for the child to judge. The sets were presented in random order. Each set contained intermingled members of the two subclasses. For each set, the child selected one toy at a time for testing. Each toy was tested immediately after selection. The child tested the toys on three trials, and the experimenter tested them on the remaining two trials for reasons of sanitation (in the case of the whistles) and skill (in the case of the yoyos). The experimenter ended the trial after the fifth choice by announcing, "No more time."

\section{Results}

The children's initial selections of two toys from the two different subsets were considered preference for diverse evidence. The children made diverse choices; their initial two selections were from different subsets on $75 \%$ of the trials (Table 1). This was significantly different from 
chance $\left(.54,{ }^{1} p<.05\right)$. There were no significant order effects, no differences among the stimulus sets, no differences between experimenter-tested toys and child-tested toys, and no differences between the sexes.

There are other possible reasons for a child to make a sequence of diverse choices in this experiment. The most obvious one is that the demonstration, in which the two subsets differed, taught the children that subsets differ and that members of each subset must be tested in order to evaluate a kind of toy. In the next experiment, we used a different demonstration to avoid the implication that subsets differ. Subsequent studies were designed to eliminate other possible explanations of the diversity finding in Experiment 1.

In Experiment 2, we included two additional age groups: adults and 3-year-olds. Adults are known to select diverse evidence to test an inductive inference (López, 1995). If adults select diverse evidence in our task as well, that lends validity to our use of this procedure to evaluate a preference for diverse evidence in preschool children. Three-year-olds were included to explore the age limits on a preference for diverse evidence. We also provided a few defective objects as a further test of the validity of the task. If children are indeed sampling the subsets in an attempt to decide if a given type of toy is a good party favor, on subsequent tests they should focus on objects similar to the defective toy in an attempt to determine whether the defective toy is a fluke or characteristic of a subset of that kind of toy.

\section{EXPERIMENT 2}

The task of Experiment 2 was similar to that of Experiment 1 except that we (1) changed the demonstration to avoid the suggestion that subsets differ, (2) included 3 -year-olds and adults in the experiment as well as 4-yearolds, and (3) introduced defective objects to better determine the basis of the children's choices.

\section{Method}

Subjects. The subjects were twenty-four 3-year-olds (12 girls and 12 boys; age range, $3 ; 6-4 ; 0$ years, $M=3 ; 9$ ), twenty-four 4-yearolds ( 11 girls and 13 boys; age range, 4;3-4;7 years, $M=4 ; 5$ ), and 12 mothers of school-age or younger children. All the children attended a preschool serving a middle-class population. None had participated in the previous experiment.

Table 1

Mean Number of Diversity Choices by Experiment and by Age, With Standard Deviations, and Significance of Difference From Chance

\begin{tabular}{lccccl}
\hline \multicolumn{1}{c}{ Subjects } & $n$ & \multicolumn{1}{c}{$M^{*}$} & $S D$ & $t$ & $p$ \\
\hline & \multicolumn{5}{c}{ Experiment 1 } \\
4-year-olds & 12 & 3.7 & 1.5 & 2.50 & $<.05$ \\
& \multicolumn{5}{c}{ Experiment 2 } \\
3-year-olds & 24 & 2.8 & 1.1 & & $<1$ \\
4-year-olds & 24 & 4.2 & 1.0 & 7.5 & $<.001$ \\
Adults & 12 & 3.6 & 0.9 & 3.9 & $<.01$ \\
\hline
\end{tabular}

${ }^{*}$ By chance, 2.63 choices should be diversity choices.
Procedure. The experimenter modeled the task by testing four horns in random order: a defective red one that made no noise, and a red one, a green one, and a blue one that worked. The defective horn was described as "not a good party favor," and each of the others was described as "a good party favor." Then, the experimenter explained that it would not be good to buy horns as party favors.

The stimulus sets were those used in Experiment 1. However, one set of toys (the clickers) included 5 felt-decorated clickers that were disabled, 5 felt-decorated clickers that worked, and 10 wooldecorated clickers that worked. The defective clickers made up only part of a subset to avoid suggesting to the children that subsets differ. All other toys worked properly. The set of clickers was the third or fourth set presented. Otherwise, the procedure was the same as that of Experiment 1.

The adults were told that the task had been used with children; otherwise, the procedure used with the adults was the same as that used with the children.

\section{Results}

Again, the initial selection of two toys from different subsets was considered a preference for diverse evidence (Table 1). As in Experiment 1, the 4-year-olds honored diversity in their selection of test objects. Their initial two selections were diverse on $84 \%$ of the trials, which is significantly greater than chance $(p<.001)$. The adult subjects also made diverse initial selections at a frequency significantly above chance $(M=72 \%, p<.01)$. However, the diverse initial selections of the 3 -year-olds, at $56 \%$, did not differ from chance. Thus, the adults selected diverse evidence in this induction task, as had those in López's (1995) evidence selection task. This suggests that our task is a valid test of the preference for diverse evidence. The poorer performance of the adults than of the 4-year-olds, although not significant, is consistent with our informal observation that adults are sometimes less attentive than children to what they know is a childish task. The indifference of the 3-year-olds to diversity will be examined further in subsequent experiments.

What was the effect of finding a defective toy? Those subjects who could make another selection after detecting a defective toy tended to choose a toy from the same subset as the defective toy. ${ }^{2}$ In contrast, the choice immediately following selection of a nondefective toy was more likely to be from a different subset (Table 2). This pattern appeared for all the subject groups. The fact that the children changed their pattern of responses and selected an identical toy after they encountered a faulty one suggests that they were not guided by a response bias to switch from trial to trial. Rather, they appeared to be asking whether a defect is an isolated fluke or a characteristic of a class. They apparently attempted to test an "all" hypothesis (e.g., All felt-decorated clickers are defective). Adults test such hypotheses by the selection of similar objects (Spellman, López, \& Smith, 1999).

Did the 3-year-olds understand the task? To some extent, they must have, because they readily selected objects and tested them. Furthermore, as a group they responded consistently when they encountered a defective toy. This indicates that some subjects could recognize subsets. Why, then, did the 3-year-olds not select diverse objects for their initial tests? Could 3-year-olds honor diversity 
Table 2

Numbers of Subjects Selecting a Same-Subset Toy More Frequently After Selection of a Defective Toy Than After Selection of a Functioning Toy in Experiment 2

\begin{tabular}{lcc} 
Subject Group & No. of Subjects & $p$ \\
\hline 3-year-olds & 8 of 8 & $<.01$ \\
4-year-olds & 11 of 12 & $<.01$ \\
Adults & 3 of 4 & \\
\hline
\end{tabular}

with a more transparent procedure? In the next experiment, we attempted to simplify the task by requiring a single selection.

Note that the results for the 4-year-olds in the first two experiments could be due to a bias to alternate their responses from the first to the second trial. To rule out this possibility, in Experiment 3 the children were asked to select for testing one of two pairs of stimuli presented simultaneously: a pair of identical objects and a pair of objects from different subclasses.

\section{EXPERIMENT 3}

The basic task of Experiment 3 was the same as that of the previous experiments - that is, to decide if a specific kind of toy would make a good party favor. However, instead of selecting individual toys to be tested one by one, the subjects were presented with two pairs of toys, a diverse pair and an identical pair, and were asked to select the pair to be tested. This removes the possibility that a bias to alternate responses accounts for the diversity findings in the first two experiments. It also reduces the evidence selection task to a single choice between a diverse pair and a similar pair.

\section{Method}

Subjects. Twelve 3-year-olds ( 6 girls and 6 boys; age range, 3;5$3 ; 10$ years, $M=3 ; 8$ ) and twelve 4-year-olds ( 6 girls and 6 boys; age range, $4 ; 6-4 ; 10$ years, $M=4 ; 8)$ participated in the experiment. All attended a preschool serving a middle-class population, and none had participated in either of the previous experiments.

Stimuli. The stimuli were the same as in Experiments 1 and 2 except that none were disabled.

Procedure. No warm-up task was given. As in the earlier experiments, the experimenter explained that the puppet wanted good toys to use as party favors and needed the child's help. The child's task was the same as in Experiment 2 except that on each trial he or she was presented with two pairs of toys. One pair consisted of two identical toys, and the other consisted of two different-looking toys of the same kind. For each type of toy, the placement and composition of the diverse pair were counterbalanced over subjects in each age group. Each child was asked to select the pair to be tested.

\section{Results}

The 4-year-olds selected the diverse pair for testing on $67 \%$ of the trials, significantly more often than chance $(50 \%)$. The 3 -year-olds selected the diverse pair on only $48 \%$ of the trials (Table 3 ). Reducing the task to a single choice did not lead to a preference for diverse evidence on the part of the 3-year-olds.
Work by Mervis and colleagues (e.g., Johnson, Scott, \& Mervis, 1997; Mervis, Johnson, \& Mervis, 1994) indicates that many 3 -year-olds are indifferent to the existence of subbasic categories. With the exception of the animals, the types of party favors we used can be considered basiclevel categories, and their subgroups can be considered subbasic categories with limited salience. In the next experiment, we attempted to heighten 3-year-olds' sensitivity to same and different subordinate categories.

\section{EXPERIMENT 4}

In the present experiment, we gave 3-year-old subjects a series of six training trials designed to highlight the subclasses of the stimuli prior to the diversity task. On each training trial, the children were given a target object and were asked to select an object that was "the same" or "different." The training trials included the stimuli used in the diversity tests. Then, the sample selection diversity tests of Experiment 3 were given.

\section{Method}

Subjects. Twenty-four 3-year-olds (11 girls and 13 boys; age range, $3 ; 2-4 ; 0$ years, $M=3 ; 8$ ) participated in the experiment. All attended a preschool serving a middle-class population, and none had participated in any of the previous experiments.

Stimuli. For the training trials and the diversity trials, the stimuli were the same five types of toys as those used in Experiments 1, 2, and 3. None were disabled. An additional type of toy-horns, two of them plain and two with a fringe- - was also used in the training trials in order to equalize the number of trials requiring same choices and different choices. In the sample selection task, the same five pairs were used as in Experiment 3.

Procedure. Training consisted of presenting a target and two choices: a toy identical to the target and a toy from a subclass other than that of the target. The child was then asked to select either a same toy or a different toy from two alternatives. Six types of objects were used. "Same" wording was used on three trials, and "different" wording was used on three trials. The specific target (e.g., a red car or a blue car), the location of the correct choice (left or right), and the wording ("same" or "different") were counterbalanced over subjects and stimuli. The order of trials was randomized over subjects.

The sample selection procedure was the same as in Experiment 3. Again, no toys were disabled.

\section{Results}

With the training procedure, the children answered correctly on $83 \%$ of the trials when asked to select a toy that

Table 3

Selection of Diverse Pairs for Test by Experiment and by Age, and Significance of Difference From Chance

\begin{tabular}{cccccc}
\hline Subjects & $n$ & $M^{*}$ & $S D$ & $t$ & $p$ \\
\hline \multicolumn{5}{c}{ Experiment 3 } \\
3-year-olds & 12 & 2.4 & 1.3 & $<1$ & \\
4-year-olds & 12 & 3.3 & 1.2 & 2.5 & $<.05$ \\
& \multicolumn{5}{c}{ Experiment 4 } \\
3-year-olds & 24 & 2.8 & 1.5 & $<1$ & \\
\hline
\end{tabular}

*By chance, 2.5 choices should be diversity choices. 
was the same as or different from the target. Even the error of consistently selecting of an identical object when asked for a different object indicated that the children could distinguish the subclasses. Of the 24 errors, $92 \%$ consisted of selection of an identical object when asked for a different object ( $p<.001$, sign test).

However, in the sample selection task, the children selected the diverse pair of objects for testing on only $56 \%$ of the trials. This is not significantly different from chance (Table 3). The children given preliminary training (in Experiment 4$)$ selected diverse pairs more often $(56 \%)$ than did those without such training (in Experiment 3: 48\%), but this difference is not significant. In brief, we have not been successful in inducing 3-year-olds to honor diversity in selecting evidence to test a hypothesis, even when the children could distinguish members of the different subclasses.

In the final experiment, we looked for evidence of a preference for diverse toys when evaluation of a hypothesis is not at issue. Such a preference, if it exists, could account for the preference for diverse evidence.

\section{EXPERIMENT 5}

In Experiment 5, we examined sequential selections of toys without a test context for both 3-and 4-year-olds. The children's task was to pack toys away in a box with separate compartments.

\section{Method}

Subjects. Twelve 3 -year-olds ( 6 girls and 6 boys; age range, $3 ; 3-$ $3 ; 10$ years, $M=3 ; 8$ ) and twelve 4-year-olds ( 6 girls and 6 boys; age range, $4 ; 7-4 ; 11$ years, $M=4 ; 9)$ participated in the experiment. All attended a preschool serving a middle-class population, and none had participated in any of the previous experiments.

Stimuli. The same five types of toys were used as in the previous experiments. Two instances of each subgroup of each type of toy (four toys for each group) were included in each trial.

Procedure. Each child was shown five separate containers of toys, each with four instances of a different type of toy, two from each subgroup. The experimenter also produced a box with five separate compartments and asked for the child's help in packing the toys in the box. The child was told that when all the toys were packed, one kind to a compartment, he or she could take out one kind and play with those toys for a while. The child's task was to decide which type of toys to pack and to choose the compartment for that type of toys, and then to hand individual toys, one by one, to the experimenter for placement in the compartment. This procedure was followed for all five kinds of toys. On each trial, the child emptied a container onto the floor and handed toys to the experimenter, who recorded the sequence in which the toys of each kind were selected by the child. After all the toys were packed, the child selected one kind to play with for a few minutes. All the children readily helped pack toys and seemed to enjoy the session.

\section{Results}

Once the first toy is selected, by chance a different-looking toy will be selected next on two thirds (67\%) of the trials. The 3-year-olds selected a different-looking toy second on $60 \%$ of the trials; the 4-year-olds did so on $68 \%$ of the trials. Neither value is significantly different from chance, nor are the two values significantly different from each other $(t<1.0$ for all comparisons). In brief, the preference of 4 -year-olds for the selection of different-looking toys is not evident when the task is unrelated to the evaluation of a hypothesis. The 3-year-olds also showed no preference for diverse objects.

\section{DISCUSSION AND SUMMARY}

Children as young as 4 years old, like adults, select diverse evidence to test a hypothesis. This sophisticated appreciation of the importance of diversity in evidence selection for inductive inferences contrasts with this age group's less consistent performance with argument strength procedures. There are a number of reasons for which 4-year-olds might respond to diversity more readily in our sample selection task than in argument strength tasks. In our task, we used 3-D objects, which are inherently more interesting than pictures; the properties were familiar, unlike those used in many forced-choice argument strength tasks; the task did not involve labels for categories but only perception of objects; and it involved only three categories of objects on each trial (one kind of toy and two perceptibly different subsets of that kind) rather than the four, five, or more categories used in argument strength procedures. Finally, our procedure involved more active participation by the children. Certainly, the child subjects enjoyed participating.

What is the basis of the diversity responses of the 4-year-olds? Does it reflect a bias that somehow predisposes them to select diverse stimuli, or does it reflect an appreciation of diversity in hypothesis testing? The results of Experiment 5 rule out a diverse object bias. Initially, we had hoped to ask preschool subjects, as López (1995) asked college students, why they had made diverse selections. We were discouraged from doing this by pilot work with twelve 4-year-olds who were asked to select and test cars for suitability as party favors and then to justify their choices. Although most of the children's first two selections were cars of different colors, no child indicated that the second choice was based on a difference from the first choice. Furthermore, the children gave odd ("Because my mommy said to") or uninformative ("Because," "Because I like red") answers. Further probes elicited no mention of diversity. In the object selection experiments, most of the children talked spontaneously while participating. They talked about school, parties, and liking specific stimuli, but they never mentioned similarities or differences among the stimuli, unlike López's adult subjects. These observations suggest that 4-year-olds may lack access to the basis of their preference for diverse evidence. However, they in no way detract from the achievement of the preschoolers in the use of a sophisticated strategy to select useful evidence to evaluate inductive inferences.

It may be possible to devise a procedure that elicits from 4-year-olds an explicit justification for the selection of diverse evidence to test an inductive inference. Fiveyear-olds readily mentioned "same" and "different" in the 
Heit and Hahn (2001) forced-choice procedure. However, another possibility is that by age 4 children are biased to examine diverse evidence when trying to understand the world, but they do not have an accessible rationale for their choice of diverse evidence. Because such a bias to examine diverse evidence does not appear in 3-year-olds, it would be important to understand the basis for the change.

What do 3-year-olds make of our evidence selection task? Our 3-year-old subjects could discriminate the subclasses we used. They readily judged stimulus objects as members of the same subclass or different subclasses (Experiment 4). Moreover, they apparently can use strategies when selecting evidence. When faced with a defective object in Experiment 2, they employed the strategy used by adults to evaluate an all-hypothesis (e.g., "All blue cars are broken") — namely, to test another blue car (Spellman et al., 1999). However, the 3-year-olds did not select diverse evidence for testing.

Why don't 3-year-olds favor diverse evidence for inductive inferences? Either shortcomings in general information processing or lack of knowledge specific to inductive inferences could be involved. We favor an information processing account that is consistent with other differences between 3- and 4-year-olds - namely, that 3-year-olds focus on the most salient aspect of a situation, whereas 4-year-olds utilize a broader range of information. By this account, in our evidence selection task the 3-year-olds focused on the affordance of the toy-for example, whether it "worked" in a manner consistent with the canonical function of its class (e.g., rolls, makes noise). They ignored other information, such as those properties of objects that determine subset membership, unless these less salient properties were brought to their attention, as by a defective toy. Hence, they were indifferent to subset membership as they made their selections of toys for testing.

A number of differences between 3- and 4-year-olds have been observed in other tasks in which 3-year-olds appear to utilize less information, especially less salient information. For instance, in the standard false-belief task, both the child and an observer see an object hidden by the experimenter. The observer goes away, and the experimenter moves the object while the child watches. Then the child is asked where the observer will look for the object. Four-year-olds predict that the observer will look where the observer last saw the object; 3-year-olds predict that the observer will look where the object actually is (Roth $\&$ Leslie, 1998). Roth and Leslie attributed the difference between 3-year-olds and 4-year-olds to the effect of the greater salience of the contents of beliefs about reality (where the object really is) than of those of other beliefs (what the observer saw) on 3-year-olds. Similarly, in an induction task, 3- and 4-year-olds differed in their abilities to use information on prior probabilities in tests of Bayesian reasoning (Sobel, Tenenbaum, \& Gopnik, 2004). When 4 -year-olds were asked to decide if a novel object possessed a property not apparent in visual inspection, their judgments reflected their knowledge of the frequency of the property in similar objects, whereas 3 -year-olds did not use their prior knowledge of frequency. Deák, Ray, and Pick (2002) compared the abilities of 3-year-olds and 4-year-olds to match depicted objects on either shape or function when the objects varied in both respects. Threeyear-olds could match only on shape, and 4-year-olds could match on either shape or function. However, 3-yearolds could judge function and assigned labels on the basis of function. In brief, in such studies 3-year-olds utilize less information than 4-year-olds even when they have the same relevant information as the 4-year-olds.

As the authors of these findings suggest, an information processing limit can account for the differences in performance between 3- and 4-year-olds. Although each of these findings, including ours, might be explained by something specific to each task, in the interest of parsimony we prefer, at the moment, an information explanation of the difference we found between 3-year-olds and 4-year-olds.

These results leave us with several questions about the role of diverse evidence in young children's induction. What changes between age 3 and age 4? Is it merely acquisition of the ability to process more information, or is there also a change in the appreciation of the value of more information? What is the status and the source of the use of diverse evidence by the 4-year-olds? Is the ability to select diverse evidence a precondition for the evaluation of such evidence in considering inductive inferences, as in argument selection tasks? We submit that the basis of the inductive sophistication of 4-year-olds deserves further investigation.

\section{CONCLUSIONS}

Children as young as 4 years old, like adults, select diverse evidence to evaluate inductive inferences. They initially select diverse objects to be tested, and they select pairs of diverse objects rather than pairs of identical objects. These preferences do not appear in contexts in which evaluation of the evidence with respect to an inductive inference is not required. Even 3-year-olds, who do not select diverse evidence, have some awareness of the task of selecting evidence. However they are indifferent to diversity in tests of inductive inferences, as they are in nontest contexts. Questions remain about the relation between evidence selection for tests of inductive inferences and the evaluation of inductive inferences as a function of the diversity of the relevant evidence.

\section{REFERENCES}

CAREY, S. (1985). Conceptual change in childhood. Cambridge, MA: MIT Press.

Choi, I., Nisbett, R. E., \& Smith, E. E. (1997). Culture, category salience, and inductive reasoning. Cognition, 65, 15-32.

Clark, H. H., \& Clark, E. V. (1977). Psychology and language. New York: Harcourt Brace Jovanovich.

DeÁK, G. O., RAY, S. D., \& Pick, A. D. (2002). Matching and naming objects by shape or function: Age and context effects in preschool children. Developmental Psychology, 38, 503-518.

Hayes, B. K., Goodhew, A., Heit, E., \& Gillan, J. (2003). The role 
of diverse instruction in conceptual change. Journal of Experimental Child Psychology, 86, 253-276.

Heit, E., \& HaHn, U. (2001). Diversity-based reasoning in children. Cognitive Psychology, 43, 243-273.

Heit, E., Hahn, U., \& Feeney, A. (2004). Defending diversity. In W. Ahn, R. L. Goldstone, B. C. Love, A. B. Markman, \& P. Wolf (Eds.), Categorization inside and outside the lab: Festschrift in honor of Douglas L. Medin (pp. 87-99). Washington, DC: American Psychological Association.

Johnson, K. E., Scott, P., \& Mervis, C. B. (1997). Development of children's understanding of basic-subordinate inclusion relations. Developmental Psychology, 33, 745-763.

Kemler Nelson, D. G., Frankenfield, A., Morris, C., \& Blair, E. (2000). Young children's use of functional information to categorize artifacts: Three factors that matter. Cognition, 77, 133-168.

Lo, Y., Sides, A., Rozelle, J., \& Osherson, D. (2002). Evidential diversity and premise probability in young children's inductive judgments. Cognitive Science, 26, 181-206.

López, A. (1995). The diversity principle in the testing of arguments. Memory \& Cognition, 23, 374-382.

López, A., Atran, S., Coley, J. D., \& Medin, D. L. (1997). The tree of life: Universal and cultural features of folkbiological taxonomies and inductions. Cognitive Psychology, 32, 251-295.

López, A., Gelman, S. A., GutheIl, G., \& Smith, E. E. (1992). The development of category-based induction. Child Development, $\mathbf{6 3}$, 1070-1090.

Medin, D. L., Coley, J. D., Storms, G., \& Hayes, B. K. (2003). A relevance theory of induction. Psychonomic Bulletin \& Review, 10, 517-532.

MERVIS, C. B., Johnson, K. E., \& Mervis, C. (1994). Acquisition of subordinate categories by 3 -year-olds: The roles of attribute salience, linguistic input, and child characteristics. Cognitive Development, 9, 211-234.

Osherson, D. N., Smith, E. E., Wilkie, O., López, A., \& Shafir, E. (1990). Category-based induction. Psychological Review, 97, 185200.

Proffitt, J. B., Coley, J. D., \& Medin, D. L. (2000). Expertise and category-based induction. Journal of Experimental Psychology: Learning, Memory, \& Cognition, 26, 811-828.

RoTH, D., \& LesLIE, A. M. (1998). Solving belief problems: Toward a task analysis. Cognition, 66, 1-31.

ShiPley, E. F., \& SHEPPERSON, B. (2004). Category-based induction in preschool children: A second look. Unpublished manuscript.

Sobel, D. M., Tenenbaum, J. B., \& Gopnik, A. (2004). Children's causal inferences from indirect evidence: Backwards blocking and Bayesian reasoning in preschoolers. Cognitive Science, 28, 303-333.

Spellman, B. A., LóPez, A., \& Smith, E. E. (1999). Hypothesis testing: Strategy selection for generalizing versus limiting hypotheses. Thinking \& Reasoning, 5, 67-91.

\section{NOTES}

1. Chance was greater than .5 because the child's selections were made without replacement.

2. Not all of the subjects had the opportunity to make a choice after testing a defective toy: Some never selected a defective toy, and some selected a defective toy only as their final choice.

(Manuscript received February 10, 2005; revision accepted for publication August 17, 2005.) 